Avendaño, D., Rojas, J., \& Beltrán-Torres, C. (2021). El reporte integrado de EPM a la luz de la gestión de impresiones: una aproximación al análisis de información gráfica en Colombia. Contaduría Universidad de Antioquia, 79, 137-159. Doi: https://doi.org/10.17533/udea.rc.n79a06

\title{
El reporte integrado de EPM a la luz de la gestión de impresiones: una aproximación al análisis de infor- mación gráfica en Colombia
}

David Andrés Avendaño Flórez Universidad Externado de Colombia

David.avendano@uexternado.edu.co

Orcid: 0000-0002-5635-6685

Johana Katerine Rojas Madero Universidad Nacional Abierta y a Distancia Johanakaterine.rojas@outlook.es

Orcid: 0000-0001-9165-6897

César Beltrán-Torres

Universidad Externado de Colombia cesar.beltran@uexternado.edu.co

Orcid: 0000-0003-4635-1298 
El Reporte Integrado de EPM a la luz de la Gestión de Impresiones: una aproximación al análisis de información gráfica en Colombia

Resumen: A lo largo de los últimos veinte años, se ha incrementado la divulgación de informes no financieros, especialmente, en las empresas pertenecientes a industrias con altos impactos ambientales y sociales. Las motivaciones de la divulgación de información ha sido objeto de estudio en empresas con deteriorada reputación y legitimidad, puesto que este tipo de informes pueden servir para transmitir a los usuarios información sesgada que logre garantizar la aceptación social a través de diversas manifestaciones por parte de la administración. En este artículo, se analizó la presencia de Gestión de Impresión (GI) en información financiera y no financiera revelada en el Reporte Integrado (RI) 2015 de Empresas Públicas de Medellín (EPM). Desde un enfoque interpretativo y usando técnicas de: selectividad, y distorsión gráfica se evaluó si la información gráfica es usada para aumentar la transparencia y claridad o por el contrario para incidir en la impresión pública de los usuarios. Se encontró que EPM no tuvo intención decidida de auto favorecerse, pues el $40 \%$ de los gráficos favorecieron a EPM, el 30\% desfavorables y otro 30\%, no presenta GI.

Palabras clave: Reporte Corporativo, Gestión de Impresión, Análisis Gráfico, Reporte Integrado.

EPM's Integrated Report in the Light of Impression Management: An Approach to Graphic Information Analysis in Colombia

Abstract: Over the last twenty years, disclosure of non-financial reports has increased, especially in companies belonging to industries with high environmental and social impacts. The motivations for information disclosure have been the subject of study in companies with deteriorated reputation and legitimacy, since this type of reports can be used to transmit biased information to users in order to ensure social acceptance through various manifestations by the management. This paper analyzes the presence of Impression Management (IM) in financial and non-financial information disclosed in the 2015 Integrated Report (IR) of Empresas Públicas de Medellín (EPM). From an interpretative approach and using selectivity and graphic distortion techniques, it was evaluated whether graphic information is used to increase transparency and clarity or, on the contrary, to influence the public perception of users. It was found that EPM did not have a decided intention to favor itself, as $40 \%$ of the graphs favored EPM, 30\% were unfavorable and another 30\% did not reveal PM.

Keywords: Corporate Reporting, Impression Management, Graphic Analysis, Integrated Reporting.

$O$ relatório Integrado da EPM à luz da Gestão de Impressões: uma aproximação à análise de informação gráfica na Colômbia

Resumo: Ao longo dos últimos vinte anos, tem se incrementado a divulgação de relatórios não financeiros, especialmente, nas empresas pertencentes a indústrias com altos impactos ambientais e sociais. As motivações da divulgação de informação tem sido objeto de estudo em empresas com deteriorada reputação e legitimidade, dado que este tipo de relatórios pode servir para transmitir aos usuários informação tendenciosa que consiga garantir a aceitação social através de diversas manifestações por parte da administração. Neste artigo, foi analisada a presença de Gestão de Impressão (GI) em informação financeira e não financeira revelada no Relatório Integrado (RI) 2015 de Empresas Públicas de Medellín (EPM). Desde um foco interpretativo e suando técnicas de: seletividade e distorção gráfica avaliou-se se a informação gráfica é usada para aumentar a transparência e clareza ou pelo contrário para incidir na impressão pública dos usuários. Encontrou-se que a EPM não teve intenção decidida de autofavorecer-se, dado que o $40 \%$ dos gráficos favoreceram a EPM, o $30 \%$ desfavoráveis e outro $30 \%$ não apresenta GI.

Palavras chave: Relatório Corporativo, Gestão de Impressão, Análise Gráfica, Relatório Integrado.

Le rapport intégré d'EPM à la lumière de la gestion des imprimés : une approche de l'analyse de l'information graphique en Colombie

Résumé: Au cours des vingt dernières années, la publication de rapports non financiers a augmenté, en particulier dans les entreprises appartenant à des secteurs à fort impact environnemental et social. Les motivations de la divulgation d'informations ont été étudiées dans les entreprises dont la réputation et la légitimité sont détériorées, car ce type de rapport peut servir à transmettre des informations biaisées aux usagers afin d'assurer l'acceptation sociale à l'aide de diverses manifestations de la direction. Dans cet article, la présence de la gestion des impressions (GI) dans les informations financières et non financières divulguées dans le rapport intégré (RI) 2015 d'Empresas Públicas de Medellín (Entreprises Publiques de Medellín-EPM) a été analysée. À partir d'une approche interprétative et au moyen des techniques de sélectivité et distorsion graphique, il a été évalué si l'information graphique est utilisée pour augmenter la transparence et la clarté ou, au contraire, pour affecter l'impression publique des usagers. Il s'est avéré que l'EPM n'avait pas l'intention de se favoriser, puisque 40\% des graphiques étaient favorables aux EPM, 30\% étaient défavorables, et 30\% autres ne présentaient pas d'IG. Mots clés: Rapports d'entreprise, gestion des imprimés, analyse graphique, rapports intégrés. 
Cont. udea (julio-septiembre, pp. 137-159. (C) Universidad de Antioquia-2021.

\title{
El Reporte Integrado de EPM a la luz de la Gestión de Impresiones: una aproximación al análisis de información gráfica en Colombia
}

\author{
David Andrés Avendaño Flórez, Johana Katerine Rojas Madero y César Beltrán-Torres \\ https://doi.org/10.17533/udea.rc.n79a06
}

Primera versión recibida en marzo de 2021 - Versión aceptada en julio de 2021

\section{Introducción}

Las empresas, en las dos últimas décadas, se han comprometido con la elaboración de informes de sostenibilidad; este tipo de informes son predominantes en las empresas de sectores de alto impacto ambiental y social (Cho et al., 2015; Marrone et al., 2020). Distintos marcos regulatorios y metodologías han surgido para la preparación y divulgación de informes de sostenibilidad; la Guía para la elaboración de Memorias de Sostenibilidad (en adelante GRI) y Reporte Integrado (en adelante RI) son las de mayor difusión; esta última con el propósito de integrar la información financiera con la información no financiera, revelando si la compañía a lo largo de un periodo generó o destruyó valor.

El incremento exponencial de los informes de sostenibilidad ha logrado capturar la atención de los investigadores del área contable. Autores como Dumay et al., (2016) afirman que los informes empresariales son una oportunidad para las organizaciones de anunciar su desempeño y sus logros económicos, ambientales, sociales y de gobierno. Estos informes también son considerados un medio para aumentar la transparencia de las organizaciones (Arena et al., 2015; Du y Yu, 2020) y una herramienta idónea para evaluar el compromiso de las empresas con la sostenibilidad y el logro de los Objetivos de Desarrollo Sostenible - ODS (Tsalis et al., 2020.

A pesar de la extendida aceptación y búsqueda de transparencia, los informes de sostenibilidad aún no superan los cuestionamientos sobre su credibilidad y comparabilidad (Boiral et al., 2019; Talbot y Boiral, 2018), así como las controversias al ser considerados un instrumento de legitimación más que mecanismos de accountability (Gray y Bebbington, 2000; Moneva et al., 2006). Es 
más, se ha identificado que los informes son usados de forma oportunista por los gerentes para tratar de cambiar la percepción que los Stakeholders tienen sobre el desempeño corporativo; a estos intentos y estrategias oportunistas se les conoce en la literatura como Gestión de Impresiones (en adelante GI) (Merkl-Davies y Brennan, 2007). Esta es una práctica que han utilizado grandes empresas, en sus informes anuales, con alto impacto social, ambiental y un gran interés público. Cho, Roberts y Patten (2010) resaltan una relación directa entre un rendimiento ambiental deficiente y un lenguaje positivo en las memorias anuales, enfocado en divulgar buenas noticias y atribuyendo los resultados positivos a las acciones corporativas internas, situación evidenciada en los reportes anuales $10-\mathrm{K}$ de ciertas corporaciones norteamericanas. Las divulgaciones corporativas se centran en resaltar los resultados favorables, opacar el rendimiento negativo y sesgar las variables de este a través de la extensión del reporte y la manera en la que se transmite el mensaje final influyendo en la percepción de los usuarios de la información.

El análisis de la literatura nos ha permitido identificar que los reportes de sostenibilidad están sesgados por los gerentes quienes buscan impresionar a su audiencia presentando mejor su desempeño u ocultar impactos a través de diferentes estrategias, haciendo que se reduzca la calidad de la información, en los artículos de (Merkl-Davies y Brennan 2007; 2011; Brennan y Merkl-Davies, 2013), proporcionan información sobre cómo las organizaciones utilizan sus divulgaciones en los intentos de transmitir representaciones favorables de ellas al interactuar con sus partes interesadas, analizando las tácticas de gestión de impresiones. Según Cho et al. (2015) los informes voluntarios corporativos parecen estar obligados a ser egoístas y sesgados (Boiral 2016; Milne y Gray 2013), lo que hace que sea difícil para cualquier parte externa evaluar el desempeño corporativo y el impacto posterior de estas actividades sobre la base de la divulgación de información corporativa. Cho et al. (2015) destacan la importancia de mantener registros públicos accesibles y comprensibles, a partir de los cuales las partes interesadas puedan recopilar más información sobre las actividades corporativas; se ha argumentado que, en algunos casos, las organizaciones se involucran en intentos de manipular las percepciones públicas para tratar de mantener su legitimidad en la sociedad (Dar, 2009; Ylönen y Laine, 2015). Dar y Roberts (2010), soportados en la teoría de Goffman de la auto-presentación, demuestran cómo el contenido y las características de presentación corporativos, son empleados por las corporaciones que operan en industrias ambientalmente sensibles, para administrar impresiones sobre su desempeño ambiental. Cho et al. (2015) concluyen que las corporaciones utilizaron sus informes de sostenibilidad para enfatizar sus preocupaciones por los problemas ambientales y para expresar su compromiso con la protección del medio ambiente. 
Esta investigación busca analizar por primera vez en Colombia los informes corporativos a través del lente de la GI; de forma específica se propone identificar el uso de la distorsión gráfica y la selectividad en el RI 2015 de Empresas Públicas de Medellín (en adelante EPM), teniendo como fundamento la Teoría de Legitimidad (en adelante TL) y la mejora en la presentación de gráficos. EPM pertenece al sector de generación de energía, agua y gas natural, con 100\% de patrimonio público que ha aportado en los últimos diez años más de 16 billones de pesos entre transferencias, regalías e impuestos a la región y al país; cuenta con prácticas de reporte adecuadas, fortalecidas a lo largo de los años por evaluaciones internas y externas. En la última década se ha visto involucrada en situaciones que han deteriorado su reputación y legitimidad por diversos proyectos sociales y el impacto ambiental generado por el desarrollo de su objeto social. Con el fin de entender la lógica, las intenciones y la estructura del proceso de revelación, se responde la siguiente pregunta de investigación: ¿Qué acciones de GI se identifican en la información financiera y no financiera del RI 2015 de EPM?

Este artículo aporta a los contadores públicos en formación, porque les permite conocer y apropiar nuevas dinámicas de análisis de los reportes corporativos y de las divulgaciones de información financiera y no financiera en las empresas colombianas, ofreciendo herramientas para identificar la GI en los reportes corporativos y orientando a mejorar la calidad, transparencia y objetividad en busca de información menos sesgada y útil a la hora de tomar decisiones. Adicionalmente, se aporta a la disciplina contable en Colombia, pues amplía el espectro de abordajes teóricos y metodológicos que han caracterizado la investigación contable colombiana; así mismo, se motiva a investigar en temas de actual interés en la literatura internacional relacionados con RI y $\mathrm{GI}$, aspectos que están en un campo emergente que necesita ser conocido en nuestro país y en nuestra región. Las investigaciones se han centrado en países como Inglaterra, Gales, China, Australia, Países Bajos y Estados Unidos (Jones, 2011). Se aporta a las empresas en su proceso de reporte y de comunicación con los Stakeholders, pues este tipo de investigaciones han ayudado a mejorar la función de la contabilidad en la sociedad, su orden y calidad de presentación, ofreciendo una visión crítica y amplia sobre la información revelada. Finalmente, para los reguladores e inversores, al analizar la información se les brinda herramientas para realizar una evaluación íntegra y soportar la toma de decisiones con base en la información gráfica.

Esta investigación tiene lugar en el contexto empresarial colombiano, donde se identificó que las empresas de energía, materiales y minería son predominantes; sin embargo, "el sector financiero es el mayor productor de informes de sostenibilidad, seguido por el sector de generación y comercialización de energía" (Gómez y Quintanilla, 2012, p. 21). Se escogió EPM por ser pionera en revelación de información financiera y no financiera en Colombia, y por estar en 
uno de los sectores que generan alto impacto ambiental (agua, energía y gas). Centrándonos en el análisis del RI 2015 de EPM publicado a mediados del año 2016; se tomó el RI 2015, puesto que la compañía contaba con mayor experiencia en la elaboración, presentación y divulgación de información.

El artículo está presentado como sigue: en la primera parte se presentan los autores más relevantes de la GI junto con los diferentes métodos, técnicas que facilitan el análisis de la información financiera y no financiera presente en los informes de gestión, haciendo énfasis en la metodología de selectividad y distorsión de medidas. Luego se aborda la metodología con la cual se analizó la información gráfica del RI de EPM, se hace énfasis en el análisis de selectividad y el Índice de Discrepancia Gráfica (en adelante GDI por sus siglas en inglés). Por último, se presentan y discuten los principales resultados y las conclusiones.

\section{GI en los Reportes Corporativos}

Goffman (1959) menciona que la Impresión Pública (en adelante IP) tiene sus orígenes en la literatura de la psicología, en la que se afirma que cuando un individuo aparece en presencia de otros, generalmente tiene alguna razón para que su actividad transmita una impresión favorable Desde un contexto empresarial, la IP se conoce como GI, la cual se relaciona con un intento de controlar y manipular la impresión de los usuarios de la información corporativa (Clatworthy y Jones, 2001, p. 311). Los administradores pueden distorsionar la percepción de su público objetivo mediante el uso de las prácticas de GI, para informar los elementos más favorables de toda la información disponible.

La Gestión de Impresión Organizacional (GIO - OIM por sus siglas en inglés) hace referencia al comportamiento o acciones que las organizaciones utilizan para construir la impresión que los usuarios de la información pueden percibir. Bansal y Kistruck (2006), citados por Sandberg y Holmlund (2015), conceptualizan la GI como una discrepancia entre las acciones sustantivas de una compañía y la representación simbólica de las acciones relevantes. Las acciones sustantivas se refieren a los cambios reales en la operación de la compañía, mientras que las representaciones simbólicas es la manera en la que una organización presenta sus acciones ante otros. Bansal y Kistruck (2006) argumentan que las empresas pueden manipular la percepción de los Stakeholders, controlando la información que se revela y la forma en la que se muestra.

A través del tiempo se han desarrollado distintos métodos para analizar el contenido de los informes corporativos, que permiten detectar la presencia o no de GI. La tabla 1 resume los métodos de análisis de contenido, aplicados en las narrativas corporativas desde un punto de vista de GI. Esto se da a través de siete enfoques incluyendo la manipulación sintáctica, la manipulación retórica, la atribución de resultados de la organización (estudios orientados significado), manipulación de la temática (estudios de forma orientada), la selectividad 
(elección / selección de número de rendimiento), efectos / presentación visual (el subrayado) y comparaciones de rendimiento (Brennan et al., 2009).

Tabla 1. Técnicas GI

\begin{tabular}{|c|c|c|}
\hline Técnica & Definición & Autores \\
\hline 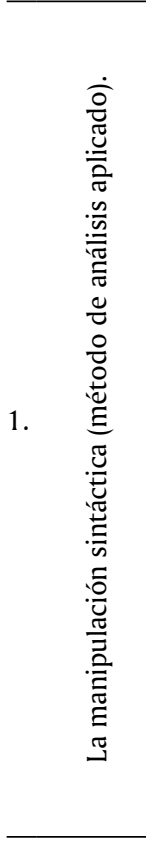 & $\begin{array}{l}\text { El mayor grupo de estudios comprende un análisis } \\
\text { del lenguaje utilizado en las revelaciones de } \\
\text { contabilidad. Gran parte de esta investigación está } \\
\text { motivada por el supuesto que los administradores } \\
\text { utilizan el lenguaje para ocultar el desempeño } \\
\text { corporativo, especialmente el rendimiento negativo. } \\
\text { Estos estudios sintácticos aplican diversos métodos } \\
\text { para medir la facilidad de lectura, centrándose en } \\
\text { el análisis de la legibilidad del texto, utilizando } \\
\text { características, tales como longitud de la oración o } \\
\text { el número de sílabas. La legibilidad es evaluada por } \\
\text { una fórmula de legibilidad que cuenta variables de } \\
\text { idioma en un texto, con el fin de proporcionar una } \\
\text { medida de la dificultad de lectura probable para los } \\
\text { lectores (Brennan et al., 2009, p. 10). }\end{array}$ & $\begin{array}{ll}1 & \text { Adelberg (1979) } \\
2 & \text { Parker (1982). } \\
3 & \text { Lewis (1986) } \\
4 & \text { Courtis (1986) } \\
5 & \text { Jones (1988) } \\
6 & \text { Baker y Kare (1992) } \\
7 & \text { Stevens et al. (1992) } \\
8 & \text { Smith y Taffler (1992) } \\
9 & \text { Subramanian (1993) } \\
10 & \text { Courtis (1995) } \\
11 & \text { Jones (1997) } \\
12 & \text { Courtis (1998) } \\
13 & \text { Sydserff y Weetman (1999) } \\
14 & \text { Clatworthy y Jones (2001) } \\
& \text { (Flesch). } \\
15 & \text { Sydserff y Weetman (2002) } \\
16 & \text { Rutherford (2003) } \\
17 & \text { Courtis (2004) } \\
18 & \text { Li (2008). } \\
19 & \text { Merkl-Davies (2007). } \\
\end{array}$ \\
\hline Técnica & Definición & Autores \\
\hline 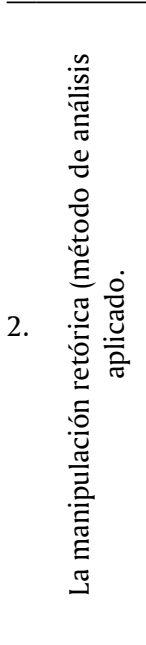 & $\begin{array}{l}\text { Esta técnica se basa en la hipótesis de la } 1 \\
\text { ofuscación, hace que la gestión de las elecciones } \\
\text { linguiísticas utilice recursos retóricos para ocultar } \\
\text { los resultados empresariales negativos. La } \\
\text { manipulación retórica implica en el ejercicio de } 2 \\
\text { opciones linguísticas la influencia de significados } \\
\text { (Brennan et al., 2009, p. 11) } \\
\text { La teórica se define como: el arte de usar el } \\
\text { lenguaje con el fin de persuadir o influir en } \\
\text { los demás; el habla o la escritura se expresan } \\
\text { en términos calculados para persuadir o } \\
\text { impresionar (a menudo en un sentido peyorativo } \\
\text { o connotación negativa de desprecio), el lenguaje } \\
\text { caracterizado por la expresión artificial u } \\
\text { ostentosa (Oxford University Press, 1989, p. 857) } \\
\text { Hasta la fecha ha habido poca investigación que } \\
\text { examine la retórica y la argumentación en los } \\
\text { informes financieros. }\end{array}$ & $\begin{array}{l}\text { Thomas (1997) (construcciones } \\
\text { pasivas, abridores de oraciones, } \\
\text { relación entre el primer y último } \\
\text { párrafo, eufemismos). } \\
\text { Jameson (2000) (múltiples voces, } \\
\text { fijación de géneros, contrastando } \\
\text { puntos focales). } \\
\text { Sydserff y Weetman (2002) [2] (índice } \\
\text { de transitoriedad, la dicción). } \\
\text { Yuthas et al. (2002) (comprensibilidad, } \\
\text { la Verdad, la legitimidad, la sinceridad, } \\
\text { la dicción). }\end{array}$ \\
\hline
\end{tabular}


El reporte integrado de EPM a la luz de la gestión de impresiones...

\begin{tabular}{|c|c|c|}
\hline Técnica & Definición & Autores \\
\hline 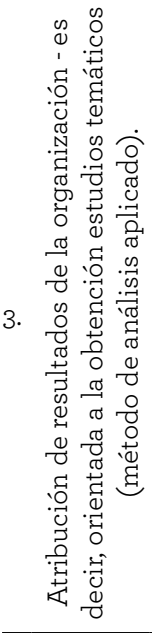 & $\begin{array}{l}\text { Esta técnica trata de investigar los patrones de } \\
\text { razonamiento causal y la atribución, utilizados para } \\
\text { explicar el desempeño corporativo. } \\
\text { Estos estudios concluyen que la administración tiene } \\
\text { una tendencia a la auto-mejora mediantela atribución de } \\
\text { responsabilidad por los resultados positivos a factores } \\
\text { internos de la organización y hacia la autoprotección } \\
\text { mediante la atribución de responsabilidad por los } \\
\text { resultados negativos a las circunstancias externas } \\
\text { (Brennan et al., 20og, p.12). } \\
\text { Por ejemplo, Frazier et al. (1984) aplica el análisis } \\
\text { factorial para extraer temas de los análisis de la gestión } \\
\text { de los resultados de las operaciones en los informes } \\
\text { anuales. }\end{array}$ & $\begin{array}{ll}1 & \text { Ingram y Frazier (1980) } \\
2 & \text { Frazier et al. (1984) } \\
3 & \text { Staw et al. (1983) } \\
4 & \text { Aerts (1994) } \\
5 & \text { Baginski et al. (2000) } \\
6 & \text { Hooghiemstra (2001) } \\
7 & \text { Aerts (2001) } \\
8 & \text { Clatworthy y Jones (2003) } \\
9 & \text { Lee et al. (2004) } \\
10 & \text { Baginski et al. (2004) } \\
11 & \text { Aerts (2005) } \\
12 & \text { Ogden y Clarke (2005) } \\
13 & \text { Barton y Mercer (2005) }\end{array}$ \\
\hline
\end{tabular}

Autores

Técnica que predominantemente gira en 1 Tennyson et al. (1990) (palabras). torno a la utilización de la administración de temas positivos y negativos, el 2 Abrahamson y Park (1994) (Las palabras clave negativas). análisis de la palabra y las frecuencias de frases con el fin de sacar conclusiones. Al igual que en otras técnicas de análisis de contenido, hay un grado de subjetividad involucrada en el análisis, ya que se basa en la clasificación de las palabras clave en categorías positivas y negativas (Brennan et al., 2009, p. 12).

Para que el método sea fiable, debe incluir una especificación correcta de medición (Jones y Shoemaker, 1994). Hasta la fecha, los estudios de análisis de contenido se han orientado de forma que investigan la gestión de impresión, y han sido relativamente simplistas; por ejemplo, mediante el conteo de palabras (Clatworthy y Jones, 2003), el número de condenas (Kohut y Cigarros, 1992), la codificación de ciertas palabras (Sydserff y Weetman , 2002). Clatworthy y Jones (2006) es más amplia, teniendo en cuenta las características textuales como información cuantitativa, variables clave de rendimiento financiero en el texto, las referencias personales, oraciones pasivas, frases orientadas al futuro.

3 Smith y Taffler (1995) (percepción del usuario).

4 Abrahamson y Amir (1996) (Las palabras clave negativas). 5 Smith y Taffler (2000) (Positivo / palabras clave negativas).

Lang y Lundholm (2000) (tipo de declaraciones 6 (rendimiento, gestión de giro, mirando hacia adelante, otros), tono de las revelaciones (optimista, pesimista)).

7 Clatworthy y Jones (2003) [5] (palabras clave positivas / negativas y declaraciones).

8 Rutherford (2005) (Las frecuencias de 90 palabras clave).

9 Davis et al. (2007) (uso Optimista Pesimista lenguaje, dicción).

Henry (2008) (palabras clave de tono (frecuencia 10 positivos / negativos - dicción)), duración de la nota de prensa, la complejidad textual, numérica de intensidad). Clatworthy y Jones (2006) (Longitud de las narrativas de contabilidad, Número oraciones pasivas, Número 11 indicadores financieros clave, Número referencias personales, referencias cuantitativas, Número futuras referencias).

12 Matsumoto et al. (2006) (palabras Número positivo tono / negativo). 


\begin{tabular}{|c|c|c|}
\hline Técnica & Definición & Autores \\
\hline 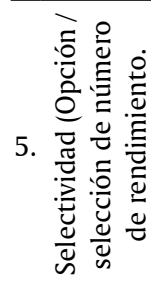 & $\begin{array}{l}\text { Consiste en la utilización de gráficos para } \\
\text { la representación de noticias relacionadas } \\
\text { con su desempeño, identificando } \\
\text { las preferencias por parte de las } 2 \\
\text { organizaciones para la presentación de } \\
\text { información, clasificándola en (i) Buena } \\
\text { Noticia, (ii) Mala Noticia o (iii) Noticia } 3 \\
\text { Neutral (Penteado, 2013, p. 34) }\end{array}$ & $\begin{array}{l}\text { Lougee y Marquardt (2004) (Pro forma } \\
\text { revelaciones de ganancias). } \\
\text { Jones: selección número de buenas y malas } \\
\text { noticias, no de rendimientos tiene una } \\
\text { asociación diferente. } \\
\text { Johnson y Schwartz (2005) (Pro forma } \\
\text { revelaciones de ganancias). }\end{array}$ \\
\hline 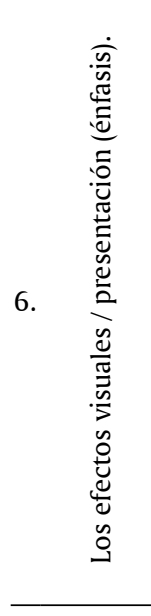 & $\begin{array}{l}\text { El discurso tiene dos elementos verbales y } \\
1 \\
\text { visuales que interactúan entre sí. Visuales } \\
\text { como gráficos, encabezados, listas } 2 \\
\text { numeradas o con viñetas, color, sombreado, } \\
\text { logotipos, pueden presagiar discusión } 3 \\
\text { verbal o reforzar los puntos claves. } \\
\text { Hay tres maneras diferentes de hacer } \\
\text { hincapié en las revelaciones en los } \\
\text { documentos narrativos de información } 5 \\
\text { financiera. En primer lugar, el énfasis } \\
\text { visual. Una segunda forma de énfasis } 6 \\
\text { es la repetición. Por último, el refuerzo } \\
\text { la información se acentúa (una palabra } 7 \\
\text { adicional para añadir énfasis a una palabra } \\
\text { clave, por ejemplo, "fuerte crecimiento" - } \\
\text { "crecimiento" es la palabra clave, "fuerte" } 8 \\
\text { es el índice (Brennan et al., 2009, p.13). }\end{array}$ & $\begin{array}{l}\text { Staw et al. (1983) (Orden de la información). } \\
\text { Courtis (1996) (Repetición). } \\
\text { Baird y Zelin (2000) (Orden de la } \\
\text { información). } \\
\text { Asi y Smith (2002) (Uso de color). } \\
\text { Courtis (2004a) (El uso del color). } \\
\text { Bowen et al.(2005) (Énfasis/posicionamiento } \\
\text { de las ganancias pro forma). } \\
\text { Kelton (2006) (Características de diseño de } \\
\text { la información contable). } \\
\text { Elliot (2006) (Énfasis / presentación de los } \\
\text { PCGA y las ganancias pro forma). }\end{array}$ \\
\hline Técnica & Definición & Autores \\
\hline 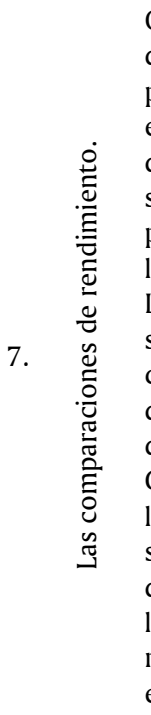 & 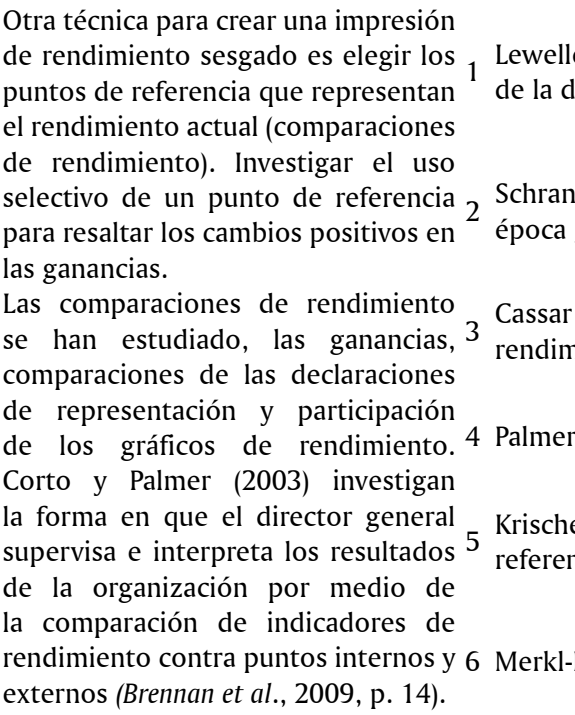 & $\begin{array}{l}\text { en et al.(1996) (Comparaciones de rendimiento } \\
\text { levolución). } \\
\text { Ad y Walther (2000) (Puntos de referencia de } \\
\text { ganancias anteriores). } \\
\text { (2001) (Divulgación de gráficos de } \\
\text { niento de acciones). } \\
\text { corta y (2003) (Referentes de rendimiento). } \\
\text { (2005) (Comparaciones Antes periodo de } \\
\text { ncia de los ingresos). } \\
\text { Davies y Brennan (2007) }\end{array}$ \\
\hline
\end{tabular}

Fuente: Elaboración propia basado en (Brennan et al., 2009). 


\section{II.1. Manipulación de gráficos en los Reportes Corporativos}

De los aspectos de GI que está recibiendo cada vez más atención es la manipulación de gráficos financieros (Jones y Beattie, 2000). La utilización de gráficos en los informes anuales corporativos se ha generalizado; el 92\% en los Estados Unidos y el $80 \%$ de las principales empresas que cotizan en bolsa utilizan gráficos (Beattie y Jones, 1997). Estos no son solo financieros, coloridos y llamativos; además, son susceptibles de ser utilizados como parte del proceso de GI.

Dos formas relevantes del uso de GI en el análisis de gráficos son: la selectividad y distorsión de medición (Jones, 2011); estas son las más utilizadas para investigar GI en los gráficos financieros y no financieros de los reportes corporativos. Según Jones y Beattie (2000) se debe examinar: (1) Variables de Rendimiento (KPV por sus siglas en inglés) (como ventas, ingresos, ganancias por acción), incluidas cuando el rendimiento ha aumentado en vez de disminuir, y (2) la distorsión de KPV en gráficos que dan una imagen menos favorable del desempeño de la compañía.

La selectividad, según Beattie y Jones (2008), consiste en la utilización de gráficos para la representación de noticias relacionadas con el desempeño societario, identificando las preferencias por parte de las organizaciones para la presentación de información, clasificándola en (i) Buena Noticia, (ii) Mala Noticia o (iii) Noticia Neutral (Penteado, 2013, p. 34). Los informes de sostenibilidad también proporcionan evidencias de selectividad, ya sea por el aumento de la información relacionada con resultados favorables o por la reducción de resultados desfavorables.

El uso de gráficos en las memorias corporativas no está libre de error (MerklDavies y Brennan, 2007). La eficacia comunicativa depende, en gran medida, de la competencia gráfica del preparador, como de la interpretación de cada usuario. Innumerables estudios documentan los incentivos y la manera en que la administración busca crear una visión más favorable del desempeño de la empresa. Esta es una motivación interesada para el uso del gráfico y las opciones de diseño de los preparadores, pues el mensaje a transmitir ya no es neutral e imparcial (Jones, 2011). Beattie y Jones (1997) describen tres formas de utilizar los gráficos comúnmente encontrados en los informes empresariales: selectividad, distorsión de la medición y mejora de la presentación.

Las organizaciones tienen libertad para determinar el contenido y la forma como presentan la información no financiera al no estar regulada (Merkl-Davies y Brennan, 2007); por tanto, aspectos en cuanto a la extensión del informe, alcance, lenguaje utilizado, tipo de palabras y/o gráficos, son los protagonistas en los que se debe centrar la investigación sobre los informes corporativos para determinar si hay uso de GI o es simplemente una extensión de revelaciones narrativas. 


\section{Metodología}

Esta investigación hace un análisis en profundidad de un grupo empresarial colombiano, en este caso EPM. Si bien los estudios sobre mejoramiento y manipulación visual tienen una naturaleza cuantitativa comparada, en el tiempo y en las organizaciones (Beattie y Jones, 2000; Jones, 2011; Merkl-Davies y Brennan, 2007); la información de EPM se obtuvo a través del sitio corporativo www.epm. com.co, en la sección de iniciativas de sostenibilidad, en la cual se evidencia la estructura del RI, según las directrices del Consejo Internacional de Reporte Integrado (en adelante IIRC por sus siglas en inglés). Se descargaron los documentos de: Nuestro Grupo, Gestión Social y Ambiental, Gestión Financiera, en los cuales se encuentra inmersa la información relacionada con el RI 2015 de EPM.

Se seleccionó el grupo EPM por pertenecer a un sector de alto impacto ambiental y, por tanto, presumiendo una necesidad creciente de legitimidad y transparencia, porque ha mostrado un compromiso con la sostenibilidad y, finalmente, por la madurez en sus procesos de comunicación y reporte corporativo. El Grupo EPM tiene su origen en EPM, empresa pública propiedad de la ciudad de Medellín. Inició su vida administrativa en enero del 1955 y es prestadora de servicios públicos domiciliarios: energía, gas natural, telecomunicaciones, agua potable, saneamiento, aseo y recolección de residuos (EPM, 2013). Hoy en día, se proyecta como un grupo empresarial multilatino conformado por 49 empresas.

Las fases en las que se desarrolló esta investigación implicaron, en primer lugar, un análisis de las características particulares presentes en los informes de sostenibilidad: (I) Madurez de los informes; con la madurez podemos establecer desde cuándo EPM está preparando sus informes de sostenibilidad y determinar que es una empresa que tiene experiencia en este tipo de reportes, adicionalmente, (II) Presencia de aseguramiento externo en los informes; es importante porque se necesita a alguien que dé su opinión y que certifique que fueron preparados de acuerdo a los establecido en el GRI; (III) Tamaño de los informes; una característica especial de las empresas con alto impacto ambiental es que sus memorias de sostenibilidad son extensas. En esta primera fase también se realizó un análisis descriptivo del contenido gráfico presente en el RI de EPM 2015.

La segunda fase corresponde a la identificación de los gráficos e información gráfica presente en el RI. Para esto se analizó el número y tipo de gráficos presentes en la información divulgada por EPM, de acuerdo con Jones (2011) se analizó el RI utilizando una lista de verificación estandarizada en donde se tenía en cuenta la frecuencia de uso de la gráfica, los temas representados, los formatos gráficos adoptados, las variables y los años graficados. Finalmente, los resultados obtenidos se comparan con los estudios de informes de sostenibilidad realizados por Jones (2011) y Penteado (2013). 
El reporte integrado de EPM a la luz de la gestión de impresiones...

La tercera y última fase involucró la aplicación de la metodología de selectividad y distorsión, para determinar el uso de la GI en el RI de EPM. Frente a la selectividad, Penteado (2013), la define como una metodología que establece si existe preferencia por parte de las organizaciones en la utilización de formas gráficas en sus informes de sostenibilidad para la presentación de información positiva. La selectividad implica clasificar la información gráfica identificada en los informes de sostenibilidad en: Buenas Noticias, Malas Noticias y Noticia Neutra, como lo muestra la tabla 2.

Una buena noticia se refiere a que la compañía se beneficia por su divulgación; por ejemplo, describiendo su trabajo con la comunidad o su labor de reciclaje. Una mala noticia se refiere a que la compañía no se beneficia por la divulgación de esta (ejemplo: presentar Incremento en emisiones de $\mathrm{CO} 2$ o usar elementos perjudiciales para el medio ambiente). Noticias Neutras la información es incomparable o no tiene variación (Penteado, 2013).

La clave para determinar si existe el manejo de las impresiones está representada gráficamente.

Tabla 2: Tendencias

\begin{tabular}{lccc}
\hline \multicolumn{1}{c}{ Naturaleza de noticia } & Tendencias exageradas & Tendencias subestimadas \\
\hline $\begin{array}{l}\text { Buenas noticias (ejemplo, aumento del reciclaje, } \\
\text { disminución de emisiones). }\end{array}$ & Favorable a la empresa & Desfavorable a la empresa \\
$\begin{array}{l}\text { Malas noticias (ejemplo, aumento de emisiones, } \\
\text { disminución en el reciclaje). }\end{array}$ & Desfavorable a la empresa & Favorable a la empresa \\
$\begin{array}{l}\text { Noticias neutras (ejemplo, datos de un año o } \\
\text { variaciones menores al 10\%) }\end{array}$ & Imparcial a la empresa & Imparcial a la empresa \\
\hline
\end{tabular}

Fuente: Tomado de (Jones, 2011).

La identificación de la selectividad se hace con base en el trabajo de Jones (2011), quien analizó 63 publicaciones de organizaciones del Reino Unido y Cho et al (2010) que analizaron 120 publicaciones de organizaciones de Estados Unidos, Reino Unido, Francia, Alemania, Italia y España.

Finalmente, para el análisis de medición de la distorsión en las gráficas, se utilizó el GDI (Tufte, 1982; Taylor y Anderson, 1986), el cual sirve para determinar el porcentaje de distorsión de la variación entre las medidas físicas presentadas en el período. Según Beattie, Dhanani y Jones (2008), cuando el gráfico no presenta ninguna distorsión, el GDI es cero. El signo del GDI, positivo o negativo, indica el porcentaje por el que la tendencia es exagerada o subestimada. Cabe resaltar que para determinar si la distorsión es favorable, desfavorable o neutra para la empresa, se debe examinar la naturaleza de la variable y la tendencia.

Existen diferentes criterios en la literatura sobre la no representatividad de las distorsiones. Penteado (2013) considera que las distorsiones son inmateriales cuando son inferiores al 5\% y Jones (2011) del 10\%. Este trabajo siguió el criterio 
establecido por Beattie y Jones (2000) para entender la manera en que las organizaciones gestionan la información gráfica en sus divulgaciones corporativas.

La fórmula del cálculo del GDI es:

$$
\text { Índice de Discrepancia Gráfica }(\mathrm{GDI})=\left(\frac{\mathrm{a}}{\mathrm{b}}-1\right)
$$

Fuente: Tomado de (Jones, 2011).

Donde "a" representa el cambio porcentual en centímetros $(\mathrm{cm})$ de cada variable y "b" representa el cambio porcentual en los datos representados en la gráfica.

La interpretación del GDI se resume en la tabla 3:

Tabla 3. Interpretación del GDI

\begin{tabular}{lcc}
\hline \multicolumn{1}{c}{ Tipo } & Favorece & Desfavorece \\
\hline Aumento del resultado favorable & GDI Positivo & GDI Negativo \\
Disminución del resultado desfavorable & GDI Positivo & GDI Negativo \\
Aumento del resultado desfavorable & GDI Negativo & GDI Positivo \\
Disminución del resultado favorable & GDI Negativo & GDI Positivo \\
\hline
\end{tabular}

Fuente: Tomado de (Penteado, 2013).

\section{Resultados}

A continuación, se detallan las características particulares presentes en los informes de sostenibilidad; analizando el Informe de EPM, teniendo en cuenta las bases establecidas por Penteado (2013), quien considera a una empresa con la madurez suficiente si lleva en promedio un tiempo de publicación de 10 años o más. EPM a 2017 publicó 14 informes de sostenibilidad desde el año 2002 y su primer RI en el año 2015, conservando varios elementos del GRI (versión G4), cumpliendo con el criterio de madurez establecido por Penteado (2013).

Para obtener una mayor credibilidad a la información publicada en los informes de sostenibilidad, según Penteado (2013), se debe revisar por parte de un experto externo. Los informes de EPM desde el año 2009 fueron preparados de acuerdo con la metodología establecida por el GRI; estos han sido certificados por entes externos, confirmando que no presentan errores significativos, incrementando su confiabilidad y validez, como lo muestra la tabla 4.

Tabla 4. Informes de sostenibilidad de EPM auditados

\begin{tabular}{clll}
\hline Año del Informe & Estados Financieros & Informes de Sostenibilidad (IS) & IS + Nivel GRI \\
\hline 2015 & Deloitte \& Touche Ltda & Deloitte \& Touche Ltda & G4. Exhaustivo \\
2014 & Deloitte \& Touche Ltda & Deloitte \& Touche Ltda & G4. Exhaustivo \\
2013 & Deloitte \& Touche Ltda & Deloitte \& Touche Ltda & G4. Exhaustivo \\
2012 & Deloitte \& Touche Ltda & Deloitte \& Touche Ltda & $3.1 \mathrm{~A}+$ \\
2011 & Deloitte \& Touche Ltda & Deloitte \& Touche Ltda & $3.1 \mathrm{~B}+$ \\
2010 & Deloitte \& Touche Ltda & ICONTEC & $3.0 \mathrm{~B}+$ \\
2009 & Deloitte \& Touche Ltda & Autodeclarado & $3.0 \mathrm{~B}$ \\
\hline
\end{tabular}

Fuente: Elaboración propia con base en (Informes de sostenibilidad EPM del año 2009 - 2015). 
El reporte integrado de EPM a la luz de la gestión de impresiones...

Otra característica especial de las empresas con alto impacto ambiental, es que sus informes de sostenibilidad son extensos (Penteado, 2013). De acuerdo con el estudio realizado por Penteado (2013), el sector que presentó los informes más extensos fue el de bienes industriales, mientras que el de bienes de consumo presentó los informes con menor número de páginas. Penteado (2013) determinó que en promedio cada uno tenía entre 136 y 324 páginas; cabe resaltar que este estudio es de 50 informes de sostenibilidad preparados bajo la estructura del GRI. A continuación, en la tabla 5 se detallan el número de páginas del RI de EPM.

Tabla 5. Número de páginas que componen cada sección RI EPM 2015

\begin{tabular}{lcc}
\hline \multicolumn{1}{c}{ Nombre del informe } & \# de páginas & \% \\
\hline Nuestro grupo & 58 & $7 \%$ \\
Gestión Social y Ambiental & 503 & $58 \%$ \\
Gestión Financiera & 309 & $36 \%$ \\
Total & 870 & $100 \%$ \\
\hline
\end{tabular}

Fuente: Elaboración propia.

Al presentar el RI, EPM revela toda la información del Grupo Empresarial, teniendo un número de páginas mayor al promedio presentado por Penteado (2013). A mayores niveles de calificación GRI (EPM GRI 4, Exhaustivo), mayor es el tamaño del informe. Adicionalmente, EPM al ser una empresa con un impacto ambiental alto, tiende a extenderse en la información a presentar.

\section{IV.1. Análisis del número de gráficos e información gráfica}

La información de EPM se compara con los estudios de informes de sostenibilidad realizados por Jones (2011), quien analizó 63 reportes corporativos de 100 empresas británicas de capitalización bursátil. Por otro lado, Penteado (2013) estudió los informes de 50 empresas brasileras que cotizan en la Bolsa de Valores de San Pablo.

En el trabajo de Jones (2011), las empresas extractivas, industriales, bebidas, tabaco, productos alimenticios y construcción, catalogadas con alto impacto ambiental, presentaron en promedio 12,3 gráficos, mientras que las compañías de bajo impacto ambiental mostraron en sus informes 9,1 gráficos.

Los informes de sostenibilidad analizados por Penteado (2013) presentaron en promedio 22,8 gráficos; donde los sectores considerados de alto impacto ambiental tienen un promedio de 43 gráficos por informe.

EPM en su RI presenta un total de 44 gráficos, esto corrobora que las empresas pertenecientes a industrias de alto impacto ambiental tienden a presentar en sus informes de sostenibilidad más gráficas que las empresas que tienen un impacto ambiental bajo. 
El análisis de número de gráficos de (Penteado, 2013) y el RI de EPM 2015 coinciden. Según Beattie y Jones (2010), los países que tienen mayor avance en términos de informes de sostenibilidad, tienden a presentar menos gráficos, comparados con los países que poco a poco van implementando estas prácticas de revelación, entre ellos los países latinoamericanos.

Para divulgar la información de manera sencilla al usuario, las empresas optan por utilizar la presentación gráfica por encima de la explicación narrativa. La diferencia que se presenta entre el número de gráficos del estudio de Jones (2011) y el RI de EPM 2015, se debe principalmente al marco utilizado para la elaboración de los informes. En EPM se utiliza la estructura propuesta por el IIRC, aplicando elementos del GRI versión G4, mientras que en la investigación de Jones (2011), la guía fue GRI.

Jones (2011) enumera las diferentes categorías de gráficos, utilizando la clasificación establecida de acuerdo con la Triple Cuenta de Resultados (en adelante TBL por sus siglas en inglés). Los gráficos se distribuyeron de la siguiente manera: $57 \%$ categoría ambiental, $25 \%$ categoría social y $18 \%$ categoría financiera. Los temas que tenían mayor número de gráficos ambientales, fueron (consumo de energía, emisiones atmosféricas, salida de residuos, uso del agua y otros, como datos de los empleados).

Penteado (2013) determina que la mayoría de la información gráfica está representada en la dimensión económica (58\%), seguido de la dimensión social $(26 \%)$ y ambiental (16\%). Cabe resaltar que los sectores de alto impacto ambiental siguieron la misma clasificación; es decir, la mayoría de los gráficos se detallaron en la dimensión financiera.

Para el análisis se decidió agrupar los diferentes capitales presentados en el RI de EPM bajo las dimensiones establecidas por el TBL (social, económico y natural) con el objetivo de hacer comparable la información. En la tabla 6 se muestra el número de gráficos según las dimensiones del TBL.

Tabla 6. Gráficos EPM por dimensiones según TBL

\begin{tabular}{lcc}
\hline Dimensión según TBL & Cantidad & $\%$ \\
\hline Social & 21 & $48 \%$ \\
Económico & 15 & $34 \%$ \\
Natural & 8 & $18 \%$ \\
Totales & 44 & $100 \%$ \\
\hline
\end{tabular}

Fuente: Elaboración propia.

Según Beattie et al. (2008), se ha establecido que las empresas de alto impacto ambiental están interesadas en hacer hincapié en sus actividades sociales y medioambientales. La información de EPM corrobora lo dicho por Beattie et 
al. (2008), puesto que, la dimensión ambiental y social, en su conjunto utilizaron más gráficos (66\% de los gráficos) que la dimensión económica (34\%).

El estudio de Penteado (2013) y el RI de EPM coinciden en mostrar un mayor número de gráficos en las dimensiones sociales y financieras, dejando la dimensión ambiental como un tema poco relevante (Jones, 2011).

En cuanto al tipo de gráfico, el más utilizado es el de columna; este dato se asemeja al expuesto por Jones (2011) y Penteado (2013). Por lo general los gráficos, principalmente los de columnas, son capaces de interpretar esta información de manera sencilla y eficaz (Beattie y Jones, 2010). En el caso de EPM, evidenciamos que los tipos de gráficos utilizados fueron: Columnas 79,5\% (35 gráficas), Tortas $15,9 \%$ (7 gráficas) y Líneas $4,5 \%$ (2 gráficas).

IV.1.1. Número de gráficos e información gráfica (Jones, 2011) vs (EPM RI, 2015)

Jones (2011) plantea que las empresas tienden a graficar un año de información, debido a que, si muestran información comparativa por más de un año, tienen mayor riesgo a presentar una imagen menos favorable de la compañía.

En el estudio de EPM el $57 \%$ de los gráficos representan solo un periodo de un año, mientras que el $43 \%$ representan gráficos que cubren de 2 a 4 años. Es pertinente aclarar que no se muestran gráficos que cubran 5 o más años.

\section{IV.1.2. Número de gráficos e información gráfica (Penteado, 2013) vs (EPM RI, 2015)}

Para el análisis de la información gráfica, Penteado (2013), plantea un análisis de posicionamiento de la misma, que se determina en función de la página en la que presenta frente al número total de páginas del informe (Penteado, 2013).

Tabla 7. Distribución de gráficas en el RI EPM 2015

\begin{tabular}{lcccc}
\hline Concepto & Intervalo Inicial & Intervalo Final & $\mathrm{N}$ & $\%$ \\
\hline (i) Inicio & 1 & 218 & 1 & $2 \%$ \\
(ii) Principio-medio & 219 & 435 & 43 & $98 \%$ \\
(iii) Medio final & 436 & 653 & 0 & $0 \%$ \\
(iv) Final, & 654 & 870 & 0 & $0 \%$ \\
Total & & & 44 & $100 \%$ \\
\hline
\end{tabular}

Fuente: Elaboración propia.

Según Beattie y Jones (2010) las empresas de alto impacto ambiental tienden a presentar la mayoría de sus gráficos en las primeras dos partes del documento. Esta tendencia aumenta cuando la información representa un resultado favorable para la compañía. Las empresas ubican la mayoría de sus gráficos en la parte inicial para llamar la atención de los usuarios quienes se concentran en las primeras páginas del informe. 


\section{IV.2. Selectividad en el informe de EPM}

En el RI se identifica la posible preferencia por parte de EPM en la utilización de formas gráficas para la presentación de información positiva en relación con su desempeño.

En EPM se identifica que un $77 \%$ de las gráficas se refieren a Buenas Noticias (34 gráficas), $20 \%$ equivalen a Malas Noticias (9 gráficas) y el 3\% son Noticias Neutras (1 gráfico). Este resultado se relaciona con los trabajos de Jones (2011) y Cho et al. (2010), quienes identificaron que el 75\% de los gráficos utilizados en informes de sostenibilidad representan buenas noticias para las organizaciones, ya sea por el aumento de resultados favorables o por la reducción de resultados desfavorables. Las empresas de industrias de alto impacto tienden a presentar más buenas noticias que malas noticias en sus gráficos y distorsionar los gráficos favorables.

\section{IV.3. GDI en el RI de EPM}

Con el objetivo de determinar si los gráficos del informe de EPM han sido manipulados en las medidas físicas para verse favorecidos o desfavorecidos, analizamos los resultados sobre el GDI, junto con la selectividad, identificando las intenciones de EPM al presentar gráficos en sus informes de sostenibilidad.

Gráfico 1. Salario básico promedio mes.

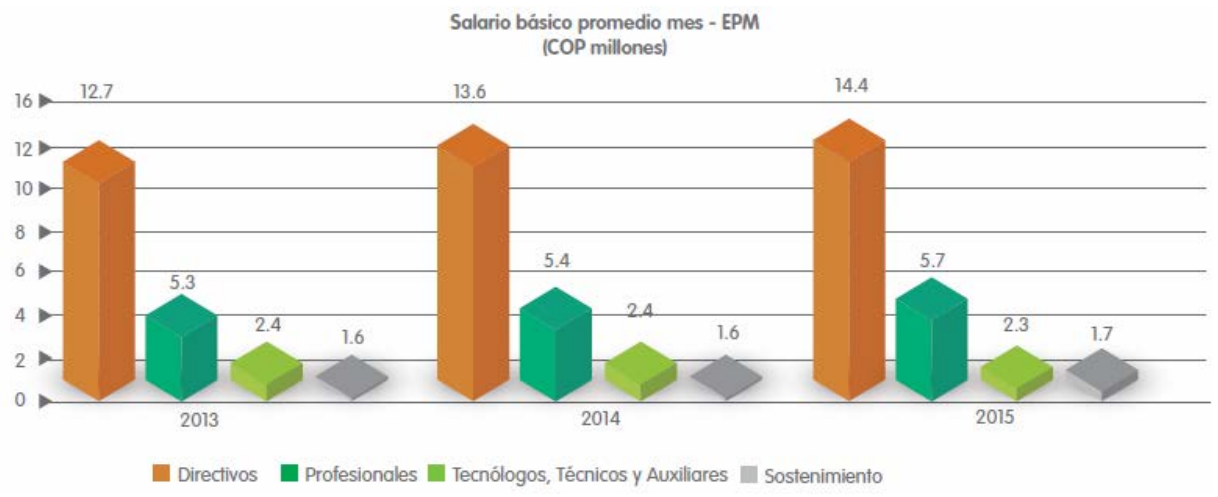

Fuente: tomado de (EPM, 2015).

El gráfico Salario básico promedio mes de EPM, es una buena noticia con cuatro variables (Directivos, Profesionales, Tecnólogos, Técnicos, Auxiliares y Sostenimiento) de tendencia creciente durante los tres años, con GDI superior al $10 \%$ en todas sus variables.

Se esperaría que EPM presente el gráfico con tendencia creciente; es decir, un GDI positivo con un porcentaje alto y dar una imagen favorable sobre la composición salarial. 
El reporte integrado de EPM a la luz de la gestión de impresiones...

Como resultado del análisis de gráficos tenemos que las cuatro variables son crecientes y el GDI esta dado así: $-15 \%$ Directivos, $+184 \%$ Profesionales, $+243 \%$ Tecnólogos, Técnicos y Auxiliares y $+220 \%$ Sostenimiento.

Se evidencia que, en la variable de compensación a Directivos, la altura de la columna está un poco alejada de la medida correcta, por lo cual EPM se vio desfavorecida de GI, dado que el salario de 2013 es de COP 12,7 millones con un aumento de aproximadamente COP 2 millones en 2015. Para los Directivos, este incremento es superior a la composición salarial que EPM ofrece a los demás grupos ocupacionales (Profesionales [\$400.000], Tecnólogos [\$-100.000] y Sostenimiento [\$100.000]), por lo cual se esperaba que EPM se favoreciera de GI.

En las tres variables: Profesionales, Tecnólogos, Técnicos y Auxiliares y Sostenimiento, se exagera el diseño de las columnas confirmando así la presencia de GI y la impresión pública.

Gráfico 2. Residuos generados EPM.

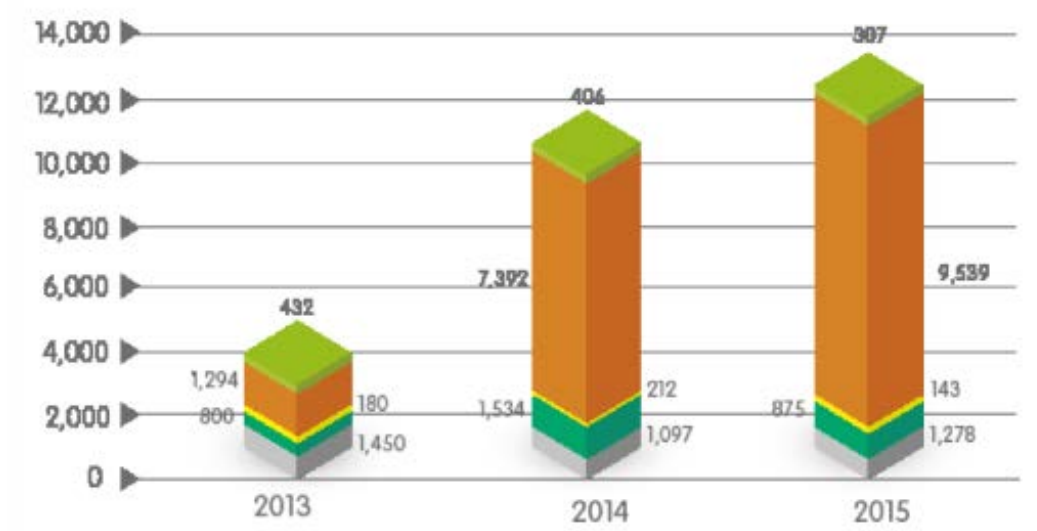

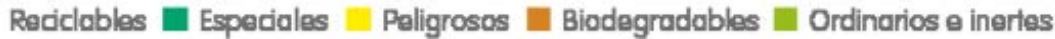

Fuente: tomado de (EPM, 2015).

El gráfico Residuos generados por EPM, es una mala noticia con cinco variables (Reciclables, Especiales, Peligrosas, Biodegradables y Ordinarios e inertes), las cuales presentan tendencia mixta, y un GDI superior al 10\% en cuatro de las variables. La variable Biodegradables, presenta un índice inferior al $10 \%$ por lo tanto no presenta distorsión en los datos.

Se esperaría que EPM, para verse favorecida de GI, debería disminuir las variables presentadas, buscando obtener un GDI negativo, tratando de hacer pequeña la altura de las columnas.

En el gráfico, la variable de tendencia creciente es Residuos Especiales con GDI $+167 \%$; por lo tanto, el desempeño de EPM se ve desfavorecido, ya que las 
acciones que ha tomado para la prevención de generación de residuos especiales tales como aparatos eléctricos y electrónicos, escombros, llantas y lodos no peligrosos, ha incrementado en 2013 , en el cual generaba 800 toneladas, mientras que en 2015 generó 875 toneladas.

Las variables de tendencia decreciente con GDI positivo se presentan en la variable Reciclables con un resultado de $+181 \%$. Esto es evidencia suficiente para afirmar que existe presencia de GI ya que el GDI es superior al $10 \%$; sin embargo, no se favorece EPM, dado que es GDI positivo en una "disminución del resultado favorable".

Por otro lado, se disminuye la generación de residuos peligrosos. En 2013 se generaban 180 toneladas y, en 2015, 143 toneladas; debido a los proyectos implementados por EPM para el manejo de sustancias peligrosas. La variable tiene un GDI $-51 \%$, en la que se evidencia la presencia de GI en el gráfico donde EPM se ve favorecida en su uso, debido a que la medida de la columna no presenta la altura correcta.

\section{Conclusiones}

Esta investigación identificó que la información gráfica, en especial sobre aspectos sociales y ambientales, se utilizan de forma extendida en el RI del EPM. Esto refleja que la información gráfica es fundamental en las divulgaciones financieras y sobre todo no financieras; es decir, es un método importante y valioso de comunicación social y ambiental corporativa. En línea con Davison (2015), las formas visuales son importantes para la contabilidad, debido a su poder y su ubicuidad en una sociedad cada vez más visual. Sin embargo, la ausencia de trabajos previos, refleja un descuido de la comunidad por un asunto tan relevante, pero erróneamente calificado como decorativo (García-Sánchez y Araújo-Bernardo, 2020).

Después de aplicar la técnica de análisis de gráficos (selectividad y distorsión de medidas físicas), se puede afirmar que en el RI de EPM 2015 existe presencia de GI, especialmente en la información no financiera. La información financiera se presentó en su mayoría mediante narrativas o tablas y no en gráficos. EPM con la información gráfica busca reducir el riesgo a presentar una imagen menos favorable ante los Stakeholders, toda vez que el $43 \%$ de los gráficos del RI de EPM, presenta información comparativa de dos a cuatro años, mientras que el $57 \%$ ilustra un solo año.

Desde la perspectiva de la GI se concluye que EPM utilizó los gráficos para distorsionar la percepción de los usuarios en el 70\% de las variables del RI de EPM 2015; esto se corroboró a través del cálculo del Índice GDI, el cual fue superior al $\pm 10 \%$. Sin embargo, no se evidencia una intencionalidad de EPM para favorecerse por medio de la GI; un $40 \%$ de las variables favorece, el 30\% desfavo- 
rece y el $30 \%$ restante de las variables fue imparcial para la IP de los usuarios del RI de EPM (la variación no es significativa).

Esta investigación presenta varias limitaciones; la primera, es sobre el número de empresas y sectores sobre los que se hizo el análisis gráfico; es necesario ampliar los años analizados, el número de reportes e incluir sectores que impactan y los que no impactan; la segunda, incorporar sotfware para análisis de gráficos que permitan una mayor agilidad, amplitud y replicabilidad; finalmente, el análisis se realiza con base en la información gráfica contenida en el RI de EPM 2015; sin embargo, se hubiera podido ampliar más la comprensión del uso e intencionalidad si se hubieran realizado entrevistas con las áreas encargadas de la preparación y presentación de la información en EPM.

Este es el primer estudio sobre esta área emergente en Colombia, por lo tanto sería muy útil si, para futuras investigaciones, se incorporan más reportes, más sectores con diferentes niveles de impacto y analizando los resultados a nivel de enfoques económicos, sociales y ambientales, y de esta forma encontrar más evidencia de GI en la información gráfica (es decir, más gráficos, tendencias más favorables y distorsiones más favorables), en especial por parte de empresas de alto impacto. Así también, se invita a realizar futuras comparaciones entre países de la región y en países donde ya hay evidencia empírica sobre la presencia y utilidad de la información gráfica. Es necesario hacer estudios longitudinales, pues solo así se evalúan las prácticas de reporte a lo largo del tiempo y las determinantes para el uso discrecional de información gráfica en la información no financiera; finalmente, se sugiere para investigaciones futuras analizar la presencia de GI desde las técnicas de análisis de contenido (presentación visual, temática, retorica, atribución de resultados), ya que EPM presenta numerosa información en tablas. Adicionalmente, se sugiere comparar los resultados con diferentes empresas colombianas que reporten bajo los criterios del RI, con el objetivo de identificar las diferencias que se presentan cuando las empresas reportaron bajo los lineamientos del GRI y las directrices del IIRC.

\section{Referencias bibliográficas}

Arena, C., Bozzolan, S. \& Michelon, G. (2015). Environmental Reporting: Transparency to Stakeholders or Stakeholder Manipulation? An Analysis of Disclosure Tone and the Role of the Board of Directors. Corporate Social Responsibility and Environmental Management, 22(6), 346-361. https://doi.org/10.1002/csr.1350

Bansal, P., Kistruck, G. \& Seeing, I. (Not) Believing: Managing the Impressions of the Firm's Commitment to the Natural Environment. J Bus Ethics 67, 165-180 (2006). https:// doi.org/10.1007/s10551-006-9021-9

Beattie, V. \& Jones, M. (1997). Visual Perception of Financial Information in Corporate Reports: The Role of Graphs. Discussion Paper No. 97/01, University of Stirling. 
Beattie, V., \& Jones, M. (2000). Impression management: The case of inter-country financial graphs. Journal of International Accounting, Auditing and Taxation, 9(2), 159-183. https://doi.org/10.1016/S1061-9518(00)00030-6

Beattie, V., Dhanani, A. \& Jones, M. (2008). Investigating Presentational Change in U.K. Annual Reports A Longitudinal Perspective. Journal of Business Communication. 45. 10.1177/0021943607313993.

Boiral, O., Heras-Saizarbitoria, I. \& Brotherton, M. (2019). Assessing and Improving the Quality of Sustainability Reports: The Auditors' Perspective. Journal of Business Ethics, 155(3), 703-721. https://doi.org/10.1007/s10551-017-3516-4

Brennan, N. M., Guillamon-Saorin, E. \& Pierce, A. (2009). Methodological Insights: Impression management\&colon; Developing and illustrating a scheme of analysis for narrative disclosures - a methodological note. Accounting, Auditing \& Accountabiy Journallit, 22(5), 789-832. https://doi.org/10.1108/09513570910966379

Clatworthy, M. \& Jones, M. (2001). The Effect of Thematic Structure on the Variability of Annual Report Readability. Accounting Auditing \& Accountability Journal. 14. 311. 10.1108/09513570110399890.

Cho, C. H., Laine, M., Roberts, R. W. \& Rodrigue, M. (2015). Organized hypocrisy, organizational façades, and sustainability reporting. Accounting, Organizations and Society, 40, 78-94. https://doi.org/10.1016/j.aos.2014.12.003

Cho, Ch., Roberts, Patten, D. (2010). 'The Language of US Corporate Environmental Disclosure'. Accounting, Organizations and Society. 35. 431-443. 10.1016/j. aos.2009.10.002.

Davison, J. (2015). Visualising accounting: An interdisciplinary review and synthesis. Accounting and Business Research, 45(2), 121-165. https://doi.org/10.1080/000147 88.2014.987203

Du, S. \& Yu, K. (2020). Do Corporate Social Responsibility Reports Convey Value Relevant Information? Evidence from Report Readability and Tone. Journal of Business Ethics, 0123456789. https://doi.org/10.1007/s10551-020-04496-3

Dumay, J., Bernardi, C., Guthrie, J. \& Demartini, P. (2016). Integrated reporting: A structured literature review. Accounting Forum, 40(3), 166-185. https://doi.org/10.1016/j. accfor.2016.06.001

EPM. (2013). Historia, antecedentes y logros del Grupo EPM.

EPM. (2015). Gestión social y ambiental.

EPM. (2015b). Gestión social y ambiental. Medellín. http://www.sostenibilidadgrupoepm. com.co/wp-content/uploads/2016/06/Gestion_Social_y_Ambiental.pdf

EPM. (2015c). Informe de Gobierno Corporativo. Medellín. http://www. sostenibilidadgrupoepm.com.co/wp-content/uploads/2016/04/informe-gobiernocorporativo.pdf

EPM. (2015d). Nuestras cifras | Informe de Sostenibilidad EPM 2015. http://2015. sostenibilidadgrupoepm.com.co/nuestro-grupo/nuestras-cifras/

EPM. (2015e). Nuestro Grupo. Medellín. http://www.sostenibilidadgrupoepm.com.co/wpcontent/uploads/2016/04/Nuestro-Grupo.pdf

Contaduría Universidad de Antioquia - No. 79. Medellín, julio-diciembre de 2021 
El reporte integrado de EPM a la luz de la gestión de impresiones...

EPM. (2016). Quienes somos EPM 2016. Medellín. http://www.epm.com.co/site/Portals/0/ Institucional/quienes-somos-2016.pdf

EPM. (2017a). Comunicación directa y presencial. http://www.epm.com.co/site/ comunidadymedioambiente/Comunidadymedioambiente/Comunidad/ Comunicacióndedoblevía/Comunicacióndirecta.aspx

EPM. (2017b). Energia para un futuro sostenible. In $9^{\circ}$ Encuentro RSE Grupo EPM. http:// www.epm.com.co/site/home/sostenibilidadepm.aspx

EPM. (2017c). Macrotendencias | Informe de Sostenibilidad EPM 2016. http://www. sostenibilidadgrupoepm.com.co/nuestro-grupo/nuestros-retos/macrotendencias/

EPM. (2017d). Medios de comunicación externa. http://www.epm.com.co/site/Home/ MediosdecomunicacionEPM.aspx

EPM. (2017e). Medios y espacios de comunicación. http:/www.epm.com.co/ site/comunidadymedioambiente/Comunidadymedioambiente/Comunidad/ Comunicacióndedoblevía/Espaciosdecomunicación.aspx

EPM. (2017f). Relacionamiento con medios alternativos, ciudadanos y comunitarios de comunicación. http:/www.epm.com.co/site/comunidadymedioambiente/ Comunidadymedioambiente/Comunidad/Comunicacióndedoblevía/ Relaciónconmedios.aspx

Goffman, E. (1959). The presentation of self in everyday life (Penguin Books (ed.)).

Gómez Villegas, M. y Quintanilla, D. (2012). Los informes de responsabilidad social empresarial: su evolución y tendencias en el contexto internacional y colombiano. Cuadernos de Contabilidad, 13(32), 121-158.

Gray, R. \& Bebbington, J. (2000). Environmental Accounting, Managerialism and Sustainability: Is the Planet Safe in the Hands of Business and Accounting ? In Advances in Environmental Accounting \& Management (Vol. 1, pp. 1-44). Emerald Group Publishing Limited. https:/www.emeraldinsight.com/doi/pdfplus/10.1016/ S1479-3598\%2800\%2901004-9

Jones, M. J. \& Beattie, V. B. (2000). Impression Management: the case of inter country financial graphs. Journal of International Accounting, Auditing \& Taxation, 9(2), 25.

Jones, M. (2011). The nature, use and impression management of graphs in social and environmental accounting. Accounting Forum, 35(2), 75-89. https://doi.org/10.1016/j. accfor.2011.03.002

Marrone, M., Linnenluecke, M., Richardson, G. \& Smith, T. (2020). Trends in environmental accounting research within and outside of the accounting discipline. Accounting, Auditing \& Accountability Journal, 33(8), 2167-2193. https://doi.org/10.1108/AAAJ03-2020-4457

Merkl-Davies, D. M. \& Brennan, N. M. (2007). Discretionary Disclosure Strategies in Corporate Narratives: Incremental Information or Impression Management? Journal of Accounting Literature, 27, 116-194. https://doi.org/http://dx.doi.org/10.2139/

Moneva, J., Archel, P. \& Correa, C. (2006). GRI and the camouflaging of corporate unsustainability. Accounting Forum, 30(2), 121-137. https://doi.org/10.1016/j. accfor.2006.02.001 
Penteado, I.. (2013). Gerenciamento de impressão em relatórios de sustentabilidade no Brasil: Uma análise do uso de gráficos. Universidad de São Paulo.

Sandberg, M. \& Holmlund, M. (2015). Impression Management Tactics in Sustainability Reporting. Social Responsibility Journal, 11(4), 677-689. https://doi.org/10.1108/SRJ-122013-0152

Talbot, D. \& Boiral, O. (2018). GHG Reporting and Impression Management: An Assessment of Sustainability Reports from the Energy Sector. Journal of Business Ethics, 147(2), 367-383. https://doi.org/10.1007/s10551-015-2979-4

Tsalis, T., Malamateniou, K., Koulouriotis, D. \& Nikolaou, I. (2020). New challenges for corporate sustainability reporting: United Nations' 2030 Agenda for sustainable development and the sustainable development goals. Corporate Social Responsibility and Environmental Management, December 2019, 1617-1629. https://doi. org/10.1002/csr.1910 